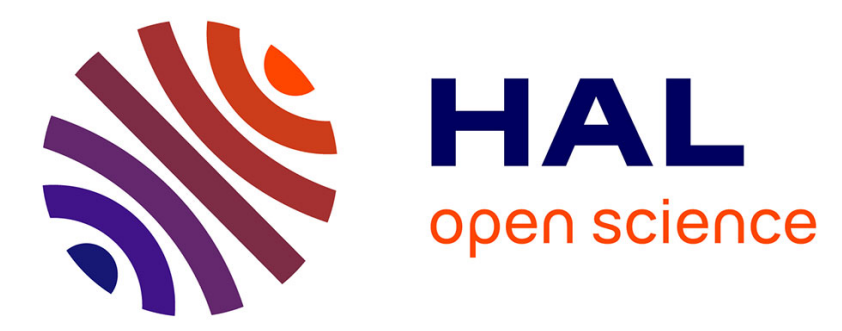

\title{
Quelle place pour la recherche des anticorps antiphosphatidyléthanolamine dans la suspicion du syndrome des antiphospholipides séronégatif?
}

P. Billoir, S. Miranda, J. Abboud, G. Armengol, H. Levesque, Y. Benhamou, V. Le Cam Duchez

\section{To cite this version:}

P. Billoir, S. Miranda, J. Abboud, G. Armengol, H. Levesque, et al.. Quelle place pour la recherche des anticorps antiphosphatidyléthanolamine dans la suspicion du syndrome des antiphospholipides séronégatif?. La Revue de Médecine Interne, 2019, 40, pp.351 - 354. 10.1016/j.revmed.2018.11.013 . hal-03484945

\section{HAL Id: hal-03484945 \\ https://hal.science/hal-03484945}

Submitted on 20 Dec 2021

HAL is a multi-disciplinary open access archive for the deposit and dissemination of scientific research documents, whether they are published or not. The documents may come from teaching and research institutions in France or abroad, or from public or private research centers.
L'archive ouverte pluridisciplinaire HAL, est destinée au dépôt et à la diffusion de documents scientifiques de niveau recherche, publiés ou non, émanant des établissements d'enseignement et de recherche français ou étrangers, des laboratoires publics ou privés.

\section{(ㄷ)(1) $\$$}

Distributed under a Creative Commons Attribution - NonCommerciall 4.0 International 


\section{Quelle place pour la recherche des anticorps antiphosphatidyléthanolamine dans la suspicion du syndrome des antiphospholipides séronégatif ?}

Which place of antiphosphatidylethanolamine antibodies research in seronegative antiphospholipid syndrome suspicion?

P. Billoir ${ }^{1}$, S. Miranda ${ }^{2}$, J. Abboud ${ }^{3}$, G. Armengol ${ }^{2}$, H. Levesque ${ }^{2}$, Y. Benhamou², V. Le Cam Duchez ${ }^{1}$

${ }^{1}$ Normandie Univ, UNIROUEN, INSERM U1096, Rouen University Hospital, Vascular Hemostasis Unit, F 76000 Rouen, France

2 Normandie Univ, UNIROUEN, INSERM U1096, Rouen University Hospital, Department of Internal Medicine, vascular and thrombosis unit, F 76000 Rouen, France

${ }^{3}$ Rouen University Hospital, Vascular Hemostasis Unit, F 76000 Rouen, France

Correspondence: Paul Billoir, Service d'Hématologie Biologique, Centre hospitalier universitaire Charles Nicolle, 1 rue de Germont, 76031 Rouen, France

Tel. : +33 232888129

Fax number : + 33232880951

E-mail : paul.billoir@chu-rouen.fr 
Propos: Le syndrome des antiphospholipides (SAPL) est un syndrome clinicobiologique associant une atteinte vasculaire et la persistance d'anticorps antiphospholipides (aPL). Les patients avec des manifestations cliniques évocatrices de SAPL mais sans aPL sont considérés comme "SAPL séronégatif" (SNAPS). Le but de cette étude était d'évaluer l'intérêt de la recherche d'anticorps antiphosphatidyléthanolamine (aPE) chez les patients ayant une suspicion de SNAPS.

Méthodes: II s'agit d'une étude rétrospective sur tous les patients ayant eu une suspicion de SNAPS. La recherche d'aPE a été réalisée par une méthode ELISA "maison". Cette méthode a été comparée avec un ELISA de screening global d'aPL.

Résultats : Au total, 228 patients ont été répertoriés pour une recherche de SNAPS. La recherche d'aPE a été effectuée dans $58,3 \%$ des cas pour un événement thrombotique. Parmi ces patients, 23 avaient une recherche d'aPE positive et persistante dans le temps (10\%). Quinze patients avaient eu un accident thrombotique, 6 avaient eu une complication obstétricale et 2 un événement combiné. Le test de screening des aPL n'était positif que dans 11 ce ces 23 patients $(47,8 \%)$.

Conclusion: Ces données qui sont en faveur de l'implication des aPE dans le SNAPS. Ces résultats suggèrent qu'il semble intéressant de rechercher les autres aPL non conventionnels pour améliorer le diagnostic de SNAPS.

Purpose: Antiphospholipid syndrome (APS) is a clinico-biological syndrome which associates vascular injury and persisting antiphospholipid antibodies (aPL). Patients with clinical symptoms of APS but without aPL are defined as "seronegative APS" (SNAPS). The aim of this study was to evaluate antiphosphatidylethanolamine antibody (aPE) investigation in patients with SNAPS suspicion.

Methods: This retrospective study was conducted in patients with SNAPS suspicion. A home-made enzyme-linked immunosorbent assay (ELISA) was used to search for aPE. The results of this home-made method were compared with those from a global screening ELISA.

Results: Two hundred twenty-eight patients with SNAPS suspicion were included. Among them, 58.3\% had a thrombotic event. The home-made ELISA found positive persisting aPE in 23 patients (10\%): 15 with a thrombotic event, 6 with obstetrical 
morbidity and 2 with a combined event. The global screening ELISA was positive in only 11 of these 23 patients $(47.8 \%)$.

Conclusion: These results suggest the implication of aPE in SNAPS. However, it is necessary to search for other non-conventional aPLs to improve SNAPS diagnosis.

Mots clés: syndrome des antiphospholipides seronégatif ; anticorps antiphosphatidyléthanolamine ; thrombose ; screening anticorps antiphospholipides

Key words: seronegative antiphospholipid syndrome; antiphosphatidylethanolamine antibody; thrombosis; antiphospholipid antibodies screening 


\section{Introduction}

Le syndrome des antiphospholipides (SAPL) est une maladie auto immune caractérisée par une atteinte vasculaire thrombotique et/ou une morbidité obstétricale associée à la persistance d'anticorps antiphospholipides (aPL) [1]. Le diagnostic biologique du SAPL repose sur le dosage par des techniques immunologiques d'lgG et d'lgM anticardiolipine, anti- $\beta$-2-glycoprotéine-1, et sur la recherche d'un anticoagulant circulant de type lupique. L'un de ces éléments biologiques doit être positif deux fois dans un intervalle de12 semaines.

Cependant, le diagnostic ne peut être établi malgré la présence de symptômes cliniques évocateurs en l'absence d'un des critères biologiques. Lorsque le tableau clinique est évocateur, il est conseillé de répéter la recherche d'anticorps à distance de l'évènement du fait de la possible apparition tardive des anticorps [2]. II a été montré que, malgré l'ajout dans les critères biologiques du SAPL de la classification de Sydney, des anticorps (Ac) anti- $\beta$-2-glycoprotéine I, un faible nombre de patients présente des manifestations vasculaires associées à la présence d'aPL non conventionnels. Les patients porteurs de ces aPL, non retenus dans la classification de Sydney, sont considérés comme «SAPL séronégatif » (SNAPS) [3]. II s'agit malheureusement d'un groupe de patients qui est potentiellement sous diagnostiqué et donc probablement sous-traité ; ce qui les exposent à un risque de récidive important. Ainsi, plusieurs auteurs plaident pour l'extension du dépistage, dans ces cas difficiles, à d'autres anticorps comme les anti-annexine $\mathrm{V}$, anti-prothrombine, anti phosphatidylsérine et les antiphosphatidyléthanolamine (aPE) [4].

Parmi ces anticorps, les aPE sont associés à des évènements obstétricaux $[5,6]$ et thrombotiques [7,8]. Ces anticorps sont régulièrement retrouvés chez les patients avec lupus érythémateux systémique et manquent de spécificité dans le diagnostic de SAPL associé [9]. Actuellement, nous disposons de peu de données quant à l'intérêt diagnostic des aPE chez les patients avec un possible SNAPS.

C'est dans ce contexte que nous avons mené une étude rétrospective dont l'objectif principal est d'évaluer l'intérêt de la recherche des aPE dans une population SNAPS.

\section{Matériels et méthodes}




\subsection{Patients}

Nous avons étudié rétrospectivement toutes les demandes de recherche d'aPE entre le $1^{\mathrm{er}}$ janvier 2014 au $1^{\mathrm{er}}$ juillet 2018 au centre hospitalier universitaire de Rouen. Les recherches d'aPE sont réalisées en seconde intention, lorsque les Ac des critères de la classification de Sydney sont absents.

Les données cliniques, biologiques et les traitements ont été colligées à partir des dossiers médicaux. Chez ces patients ont été réalisées une recherche d'anticorps aPL (mélange de différents aPL pour un screening) et une recherche d'aPE.

\subsection{Screening global d'anticorps antiphospholipides}

La recherche globale d'aPL est faite par un test de type ELISA (PHOSPHO-LISAC, Theradiag, Marnes-la Valée, France). Différents PL (cardiolipine, annexine V, phosphatidylsérine, phosphatidylinositol et la phosphatidyléthanolamine) sont coatés sur une plaque et les $\lg$ ou $\operatorname{lgM}$ aPL présents dans le plasma sont mis en évidence. II s'agit d'un test global mettant en évidence des anticorps antiphospholipides sans permettre de connaître exactement leur spécificité ajouté et avec un risque de perte de sensibilité. Les valeurs seuils pour les aPL sont un taux moyen : 17,3 UA/ml pour les lgG et 16,2 UA/ml pour les lgM (99ème percentile).

\subsection{Recherche d'anticorps antiphosphatidyléthanolamine}

La recherche d'aPE a été réalisée par une méthode ELISA « maison » inspirée de la méthode de Sanmarco et al [10]. La phosphatidyléthanolamine est coatée sur une plaque puis mise contact avec le sérum ou le plasma du patient. L'utilisation d'une antiglobuline anti-IgG ou anti-IgM associée à un conjugué permet de révéler et de quantifier les IgG et IgM aPE. Les valeurs seuils pour les aPE sont: $28 \mathrm{UA} / \mathrm{ml}$ et 56 $\mathrm{UA} / \mathrm{ml}$ pour les lgG; $36 \mathrm{UA} / \mathrm{ml}$ et $72 \mathrm{UA} / \mathrm{ml}$ pour les IgM (taux moyen au 99ème percentile et taux fort au 99.9ème percentile respectivement). Ces valeurs ont été établies à partir d'échantillons de 70 plasmas témoins. Comme nos valeurs ont été établies sur plasma, la recherche d'aPE est toujours réalisée sur plasma pour éviter le risque de faux positifs sur sérum (augmentation des valeurs de 10\% par absence d'anticoagulant). 


\section{Résultats}

\subsection{Population générale}

La recherche d'anticorps différents chez des patients suspects de SNAPS a été effectuée chez 228 patients. Les causes d'exclusion étaient la présence d'un anticorps de la classification de Sydney $(n=14)$ et une recherche d'aPE pour un autre motif que pour un événement thrombotique ou obstétrical $(n=31)$. L'âge moyen est $46 \pm 16$ ans et $45,6 \%$ sont des hommes. Dans cette cohorte, le critère clinique classant en SNAPS est majoritairement un événement thromboembolique $(58,3 \%)$.

\subsection{Population aPE positive}

La présence d'aPE a été retrouvée chez 23 patients (10\%). Onze patients ont été exclus en raison d'une présence concomitante ou retardé des aPL de la classification de Sydney. Trois patients ont été exclus pour symptôme clinique non évocateur d'un SNAPS (2 troubles neurologiques non spécifiques, 1 acrosyndrome). La recherche d'aPE a principalement été faite pour une maladie thromboembolique $(n=15)$, suivi d'événements obstétricaux $(n=6)$ et 2 pour des événements mixtes (Tableau 1). Les thromboses veineuses étaient principalement des thromboses veineuses profondes (TVP) proximales $(n=9)$ ou de TVP compliquées d'embolie pulmonaire $(n=3)$ et 2 EP idiopathiques. Les thromboses artérielles étaient 2 accidents vasculaires cérébraux, 1 accident ischémique transitoire, 1 syndrome coronarien aigu et une thrombose aortique. Quatre patients avaient une pathologie sous-jacente (3 lupus érythémateux systémique, une hémopathie maligne). Trois patients ayant eu un accident thrombotique avaient une thrombophilie associée (deux mutations du factor $V$ Leiden hétérozygote, une mutation G20210A du gène de la prothrombine hétérozygote). Deux patients avaient à la fois des aPE IgG et $\lg \mathrm{M}$.

\subsection{Comparaison aPE et screening $A P L$}

Une étude a été réalisée pour évaluer si l'ELISA de screening global d'aPL global permettait de suspecter la présence d'aPE. Le screening n'a été positif que chez 11 
patients sur 23 (47,8\%). Pour 2 patients, l'isotype d'APL ne correspondait pas à l'isotype d'aPE.

\section{Discussion}

Ainsi, dans cette étude, nous retrouvons la présence d'aPE de façon persistante dans le temps chez 10\% de patient ayant une suspicion de SNAPS.

Comme pour le SAPL, la persistance des aPE semble nécessaire pour évoquer un SNAPS. Une étude réalisée chez des donneurs sains retrouvait une positivité des aPE chez $3,1 \%$ de façon transitoire [11]. Les données d'une étude rétrospective réalisé dans une série de 20 patients décrivait une disparition des aPE chez 7 patients [12]. Notre étude a été réalisée en suivant les critères de classification de Sydney, avec persistance des aPE à 12 semaines d'intervalle et recherche concomitante des aPL de la définition [1].

La prévalence d'aPE dans les études menées sur des patients qui ont présenté une TVP varie de $14,3 \%$ à 18\% [13-15]. Les aPE sont régulièrement associés aux autres APL de la classification de Sydney dans un contexte de SAPL secondaire thrombotique [13]. Dans une série de 140 patients ayant un événement thrombotique idiopathique avec suspicion de SAPL, seulement 9 patients avaient des aPE isolés et 11 avaient des Ac anticardiolipine ou des Ac anti- $\beta 2$ glycoprotéine I [14]. Nous retrouvons une présence d'aPE IgG et IgM légèrement plus importante dans notre population SNAPS thrombotique.

Le mécanisme physiopathologique de l'atteinte obstétricale par les aPE a été décrit chez l'animal [16]. II a été retrouvé une augmentation de thromboses et de cellules apoptotiques intra-placentaires. Le parallèle chez l'homme a été retrouvé dans une étude mené par Sugi et al en 1999 , avec une prévalence d'aPE IgG de $20.1 \%$ dans une série de 139 femmes incluses pour fausses couches à répétition [17]. Gris et al ont confirmé que l'isotype IgM était un facteur de risque indépendant de pertes fœtales inexpliquées, avec un odd ratio à 6 (2,3-15,7), supérieur au lupus anticoagulant et aux anti- $\beta 2$ glycoprotéine I. Les aPE étaient retrouvés comme seul aPL positif dans $73 \%$ des cas [18]. Yamada et al ont mis en évidence, sur une cohorte de 1155 femmes enceintes, un risque de développer une hypertension sévère (odd ratio : 8,3, 2,4-29) et un risque d'accouchements prématurés avant 34 semaines d'aménorrhée $(12,7,3,1-50)$ associé à la persistance d'aPE IgG [19]. 
Dans notre étude, les manifestations obstétricales étaient principalement des fausses couches précoces avec un nombre d'aPE IgG et lgM équivalent.

Les recommandations du $13^{\text {th }}$ International Congress on antiphospholipid antibodies sont une recherche d'aPE avec une technique parfaitement standardisée [4]. La littérature reste relativement peu fournie sur la recherche d'aPE et les études relativement anciennes ne sont pas conçues pour confirmer leur valeur diagnostic dans le SNAPS. Des recommandations récentes de Joste et al préconisent une recherche d'aPE en seconde intention, associée à la recherche d'antiprothrombine si présence d'une une hypothrombinémie [20]. Sciascia et al ont étudié l'association de différents aPL dans le LES, et, si les aPE étaient plus souvent présents chez ces patients, ils n'étaient pas plus fréquemment associés à des manifestations cliniques de type accident thrombotique ou obstétrical [9]. Cette étude n'a pas encore été effectuée chez les patients SNAPS et nos résultats confirment que le screening aPL ne permet pas d'orienter sur la présence d'aPE dans tous les cas. II apparait nécessaire de compléter le bilan de SNAPS par une recherche d'Ac antiphosphatidylsérine/antiprothrombine (PS/PT). Cet aPL est retrouvé chez 11,8\% des patients SNAPS, et est régulièrement retrouvé associé à un anticoagulant de type lupique $(70,4 \%)[21]$. La recherche de l'anticoagulant lupique, appartenant aux critères de classification de Sydney, peut être rendue faussement positif par la prise d'un traitement anticoagulant, particulièrement sous anticoagulant oraux directs [20]. Les anti-PS/PT seraient un marqueur de substitution du LA chez ces patients.

Les limites de notre étude sont une recherche de SNAPS en rétrospectif avec une population de patients sélectionnés et un nombre de patients limité.

\section{Conclusion}

Nous disposons de données qui sont en faveur de l'implication des aPE dans le SNAPS. II semble nécessaire de compléter la recherche de SNAPS avec les autres aPL n'appartenant pas aux critères de classification de Sydney au vu du faible pourcentage de patients positifs pour les aPE dans notre population (10\%).

\section{Conflit d'intérêts : aucun}

Remerciements: Les auteurs remercient N. Sabourin Gibbs, du Centre HospitaloUniversitaire de Rouen, pour la révision du résumé anglais. 
Référence

[1] Miyakis S, Lockshin MD, Atsumi T, Branch DW, Brey RL, Cervera R, et al. International consensus statement on an update of the classification criteria for definite antiphospholipid syndrome (APS). J Thromb Haemost JTH 2006;4:295306.

[2] Cervera R, Conti F, Doria A, laccarino L, Valesini G. Does seronegative antiphospholipid syndrome really exist? Autoimmun Rev 2012;11:581-4.

[3] Nayfe R, Uthman I, Aoun J, Saad Aldin E, Merashli M, Khamashta MA. Seronegative antiphospholipid syndrome. Rheumatol Oxf Engl 2013;52:135867.

[4] Bertolaccini ML, Amengual O, Atsumi T, Binder WL, de Laat B, Forastiero R, et al. "Non-criteria" aPL tests: report of a task force and preconference workshop at the 13th International Congress on Antiphospholipid Antibodies, Galveston, TX, USA, April 2010. Lupus 2011;20:191-205.

[5] Sugi T, Matsubayashi H, Inomo A, Dan L, Makino T. Antiphosphatidylethanolamine antibodies in recurrent early pregnancy loss and mid-to-late pregnancy loss. J Obstet Gynaecol Res 2004;30:326-32.

[6] Obayashi S, Ozaki Y, Sugi T, Kitaori T, Katano K, Suzuki S, et al. Antiphosphatidylethanolamine antibodies might not be an independent risk factor for further miscarriage in patients suffering recurrent pregnancy loss. $\mathrm{J}$ Reprod Immunol 2010;85:186-92.

[7] Sanmarco M, Gayet S, Alessi M-C, Audrain M, de Maistre E, Gris J-C, et al. Antiphosphatidylethanolamine antibodies are associated with an increased odds ratio for thrombosis. A multicenter study with the participation of the European Forum on antiphospholipid antibodies. Thromb Haemost 2007;97:949-54.

[8] Gonzales-Portillo F, Mclntyre JA, Wagenknecht DR, Williams LS, Bruno A, Biller $J$. Spectrum of antiphospholipid antibodies (aPL) in patients with cerebrovascular disease. J Stroke Cerebrovasc Dis Off J Natl Stroke Assoc 2001;10:222-6.

[9] Sciascia S, Murru V, Sanna G, Roccatello D, Khamashta MA, Bertolaccini ML. Clinical accuracy for diagnosis of antiphospholipid syndrome in systemic lupus erythematosus: evaluation of 23 possible combinations of antiphospholipid antibody specificities. J Thromb Haemost JTH 2012;10:2512-8.

[10] Sanmarco M. ELISA for antiphosphatidylethanolamine antibody detection: high impact of assay buffer on results. J Immunol Methods 2010;358:9-16.

[11] Mclntyre JA, Wagenknech DR, Waxman DW. Frequency and specificities of antiphospholipid antibodies (aPL) in volunteer blood donors. Immunobiology 2003;207:59-63.

[12] Desauw C, Hachulla E, Boumbar Y, Bouroz-Joly J, Ponard D, Arvieux J, et al. [Antiphospholipid syndrome with only antiphosphatidylethanolamine antibodies: report of 20 cases]. Rev Med Interne 2002;23:357-63.

[13] Sanmarco M, Alessi MC, Harle JR, Sapin C, Aillaud MF, Gentile S, et al. Antibodies to phosphatidylethanolamine as the only antiphospholipid antibodies found in patients with unexplained thromboses. Thromb Haemost $2001 ; 85: 800-$ 5.

[13] Hirmerova J, Ulcova-Gallova Z, Seidlerova J, Filipovsky J, Bibkova K, Micanova $Z$, et al. Laboratory evaluation of antiphospholipid antibodies in patients with venous thromboembolism. Clin Appl Thromb Off $\mathrm{J}$ Int Acad Clin Appl Thromb 2010;16:318-25. 
[15] Berard M, Chantome R, Marcelli A, Boffa MC. Antiphosphatidylethanolamine antibodies as the only antiphospholipid antibodies. I. Association with thrombosis and vascular cutaneous diseases. J Rheumatol 1996;23:1369-74.

[16] Velayuthaprabhu S, Matsubayashi H, Sugi T, Nakamura M, Ohnishi Y, Ogura T, et al. A unique preliminary study on placental apoptosis in mice with passive immunization of anti-phosphatidylethanolamine antibodies and anti-factor XII antibodies. Am J Reprod Immunol N Y N 1989 2011;66:373-84.

[17] Sugi T, Katsunuma J, Izumi S, McIntyre JA, Makino T. Prevalence and heterogeneity of antiphosphatidylethanolamine antibodies in patients with recurrent early pregnancy losses. Fertil Steril 1999;71:1060-5.

[18] Gris JC, Quéré I, Sanmarco M, Boutiere B, Mercier E, Amiral J, et al. Antiphospholipid and antiprotein syndromes in non-thrombotic, non-autoimmune women with unexplained recurrent primary early foetal loss. The Nîmes Obstetricians and Haematologists Study--NOHA. Thromb Haemost 2000;84:228-36.

[19] Yamada H, Atsumi T, Kobashi G, Ota C, Kato EH, Tsuruga N, et al. Antiphospholipid antibodies increase the risk of pregnancy-induced hypertension and adverse pregnancy outcomes. J Reprod Immunol 2009;79:188-95.

[20] Joste V, Dragon-Durey M-A, Darnige L. [Laboratory diagnosis of antiphospholipid syndrome: From criteria to practice]. Rev Med Interne 2018;39:34-41.

[21] Zohoury N, Bertolaccini ML, Rodriguez-Garcia JL, Shums Z, Ateka-Barrutia O, Sorice M, et al. Closing the Serological Gap in the Antiphospholipid Syndrome: The Value of "Non-criteria" Antiphospholipid Antibodies. J Rheumatol 2017;44:1597-602. 
Tableau 1. Caractéristiques cliniques et traitements des patients atteints d'un syndrome des antiphospholipides séronégatif à antiphosphatidyléthanolamine.

\begin{tabular}{|c|c|c|c|}
\hline & $\begin{array}{l}\text { SNAPS } \\
\text { thrombotique }\end{array}$ & SNAPS obstétrical & SNAPS mixte \\
\hline$N$ & 15 & 6 & 2 \\
\hline Age & $51,6 \pm 14,5$ & $33,2 \pm 5,1$ & 35,5 \\
\hline $\begin{array}{l}\text { Sexe } \\
\text { (homme/femme) }\end{array}$ & $6 / 9$ & $0 / 6$ & $0 / 2$ \\
\hline $\begin{array}{l}\text { Thrombose } \\
\text { veineuse profonde }\end{array}$ & 11 & & 1 \\
\hline $\begin{array}{l}\text { Thrombose } \\
\text { artérielle }\end{array}$ & 4 & & 1 \\
\hline Thrombose mixte & 1 & & \\
\hline FC précoce & & 5 & 2 \\
\hline $\mathrm{RCIU}$ & & 1 & \\
\hline \multicolumn{4}{|l|}{ Traitement } \\
\hline Anticoagulant & $\begin{array}{c}6 \text { AVK, } 2 \text { HBPM, } \\
1 \text { rivaroxaban }\end{array}$ & 1 HBPM & \\
\hline Aspirine & 4 & 1 & 2 \\
\hline SNAPS secondaire & 3 & 1 & \\
\hline aPE IgM & 6 & 3 & 2 \\
\hline Titre fort & 3 & 1 & 1 \\
\hline aPE IgG & 9 & 3 & \\
\hline Titre fort & 1 & 1 & \\
\hline
\end{tabular}

